\title{
Genetic Variation Explains Changes in Susceptibility in a Naïve Host Against an Invasive Forest Pathogen: The Case of Alder and the Phytophthora alni Complex
}

\author{
Miguel A. Redondo, ${ }^{1, \dagger}$ Jan Stenlid, ${ }^{1}$ and Jonàs Oliva ${ }^{2,3}$ \\ ${ }^{1}$ Department of Forest Mycology and Plant Pathology, Swedish University of Agricultural Sciences, Uppsala, Sweden \\ ${ }^{2}$ Department of Crop and Forest Sciences, University of Lleida, Lleida, Spain \\ 3 Joint Research Unit Agrotecnio, Forest Sciences and Technology Centre of Catalonia, Lleida, Spain \\ Accepted for publication 23 September 2019.
}

\begin{abstract}
Predicting whether naïve tree populations have the potential to adapt to exotic pathogens is necessary owing to the increasing rate of invasions. Adaptation may occur as a result of natural selection when heritable variation in terms of susceptibility exists in the naïve population. We searched for signs of selection on black alder (Alnus glutinosa) stands growing on riverbanks invaded by two pathogens differing in aggressiveness, namely, Phytophthora uniformis (PU) and Phytophthora $\times$ alni $(\mathrm{PA})$. We compared the survival and heritability measures from 72 families originating from six invaded and uninvaded (naïve) sites by performing in vitro inoculations. The results from the inoculations were used to assess the relative contribution of host genetic variation on natural selection. We found putative signs of natural selection on alder exerted by PU but not by PA. For PU, we found a higher survival in families originating from invaded sites
\end{abstract}

ABSTRACT compared with uninvaded sites. The narrow sense heritability of susceptibility to PU of uninvaded populations was significantly higher than to PA. Simulated data supported the role of heritable genetic variation on natural selection and discarded a high aggressiveness of PA decreasing the transmission rate as an alternative hypothesis for a slow natural selection. Our findings expand on previous attempts of using heritability as a predictor for the likelihood of natural adaptation of naive tree populations to invasive pathogens. Measures of genetic variation can be useful for risk assessment purposes or when managing Phytophthora invasions.

Keywords: adaptation, compartmental SIR model, disease control and pest management, ecology and epidemiology, inoculation, Phytophthora, plant-pathogen interactions, simulation
The global movement and trade of plant and soil material has facilitated the spread of plant pathogens, creating novel pathogenhost combinations, with unpredictable consequences (Budde et al. 2016; Ennos 2015; Hendry et al. 2017; Santini et al. 2013). Without a coevolutionary background, these new pathogens are expected to trigger rapid evolutionary processes in naïve hosts (Altizer et al. 2003; Carroll et al. 2014; Daszak et al. 2000). In theory, host population bottlenecks or even extinctions can be expected when new pathogens encounter hosts with small population sizes or when large inoculum reservoirs exist (De Castro and Bolker 2005; Fisher et al. 2012; McCallum 2012). High tree mortality rates have been observed following invasions by aggressive pathogens such as Cryphonectria parasitica, Ophiostoma novo-ulmi, Hymenoscyphus fraxineus, and Phytophthora ramorum, the causal agents of chestnut blight, Dutch elm disease, ash dieback, and sudden oak death, respectively (Brasier 1991; Hepting 1974; Kowalski 2006; Rizzo et al. 2005). Regardless of the aggressiveness of the pathogen, individual trees are often observed to survive the invasion (Dodd et al. 2005; Griffin 2000; Kjær et al. 2012; Solla et al. 2005). Surviving trees may carry heritable resistance that can provide adaptation potential to the future generations (Budde et al. 2016).

†Corresponding author: M. A. Redondo; miguel.angel.redondo@slu.se

Funding: This research was funded by the European BiodivERsA RESIPATH Project. J. Oliva was partly supported by a "Ramón y Cajal" fellowship (RYC2015-17459).

*The $\boldsymbol{e}$-Xtra logo stands for "electronic extra" and indicates that supplementary material, and a supplementary data file are published online.

The author(s) declare no conflict of interest.

(C) 2020 The American Phytopathological Society
However, they may also be escapers (i.e., susceptible trees that are not infected but they stand in invaded sites), in which case they will carry no breeding value.

Adaptive evolutionary responses are expected to occur when new invaders exert selection pressure on host populations of sufficient genetic size and variation (Parker and Gilbert 2004; Strauss et al. 2006). An invader exerts natural selection when (i) the invader has an effect on the host fitness, (ii) the effect on the host fitness is not random, and (iii) the offspring can inherit, at least partially, the trait on which the selection acts (Strauss et al. 2006). In extreme cases, aggressive forest pathogens may indiscriminately affect the individuals of a population. Therefore, environmental factors or other random events determine the survival of the individuals, thus impairing natural selection. Aggressiveness may also have epidemiological consequences, slowing down natural selection. As predicted by the virulence trade-off hypothesis, highly aggressive pathogens will kill trees in a very short period of time, reducing the period when they are infectious and able to produce inoculum, with a consequent decrease in the pathogen transmission rate (Alizon et al. 2009). A decrease in transmission may decrease the mortality rate and consequently the selection pressure on the host (Kroll and Eide 1981). Evaluating the relative contribution of host genetic variation in susceptibility and pathogen aggressiveness on natural selection is critical to assess the sustainability of invaded tree populations.

The Phytophthora alni-alder pathosystem, which has been responsible for the decline and mortality of alders (Alnus spp.) across Europe (Aguayo et al. 2014; Brasier et al. 2004; Husson et al. 2015; Jung and Blaschke 2004), is a good model system for studying the natural selection exerted by a newly introduced pathogen. Members of the $P$. alni complex are spread by water and are mainly restricted to riparian areas (Brasier et al. 2004). Therefore, alders growing in isolated swamps or in wet forests far 
away from the main riverbanks have not been exposed to the pathogen and can represent the alders that existed on riverbanks before the invasion. In Sweden, the first reports of the $P$. alni complex date from the 1990s (Olsson 1999). Today, P. alni damage is widespread across southern Sweden and is caused by two species: Phytophthora uniformis (PU) and Phytophthora $\times$ alni (PA) (Redondo et al. 2015). Although PA is more aggressive than PU, it is susceptible to low temperatures and long frost periods (Brasier and Kirk 2001; Černý et al. 2012). Therefore, PA is limited to coastal and southernmost areas with mild winters, whereas PU is present throughout southern Sweden (Redondo et al. 2015). As a result, there are three distinct areas of alder: areas invaded by both PA and PU, areas only invaded by PU, and uninvaded areas. Since the introduction of alder Phytophthora, $>30 \%$ of alder stands in southern Sweden have experienced mortality and decline (Redondo et al. 2015). The symptoms caused by the alder Phytophthora were more frequently found in young than in old trees (Redondo et al. 2015). This dieback of alders caused by the $P$. alni species complex can create a cascade effect on stream ecosystems (Bjelke et al. 2016). Nevertheless, at some of these sites, several trees of different ages have survived the outbreaks and show no symptoms of the disease. Whether these trees have not succumbed to disease as a result of natural selection based on susceptibility or if they have simply escaped the disease is unknown.

The aim of this study was to explore whether signs of natural selection are present in alder stands affected by PU and PA. We hypothesized that natural selection will depend on the level of preexisting genetic variation in susceptibility. Our alternative hypothesis is that selection depends on the capacity of the pathogen to cause a sufficient pressure on the host, and that this pressure decreases as a result of reduced transmission rates if pathogen aggressiveness is too high.

To examine these two scenarios, we compared the susceptibility among progenies from PA and PU invaded and uninvaded sites. Invaded sites were riverbank alder stands with high mortality, and from which the species of the $P$. alni complex were isolated during a previous survey in 2013 (Redondo et al. 2015). Uninvaded sites were healthy looking alder stands 1 to $3 \mathrm{~km}$ away from any water body and infected sites. Based on in vitro inoculations, we obtained data on survival, heritability of susceptibility among progenies from invaded and uninvaded areas by PA and PU, as well as aggressiveness values for both PA and PU. These parameters were implemented in a simple simulation integrating both selection and epidemiological aspects of the disease cycle. We evaluated whether the observed shifts in resistance could be explained by differences in genetic resistance in the original population and/or the role of the aggressiveness of the pathogen. In order to simulate selection, we used heritability of susceptibility in naive populations as an indication of the survival that occurs owing to genetic versus stochastic factors. The impact of aggressiveness on transmission rate was predicted from the decrease in the number of infectious trees via a susceptible-infected-recovered (SIR) model.

\section{MATERIALS AND METHODS}

Seed collection and germination. Alder cones were collected during October 2015 from three riverside alder stands invaded by PU, three riverside stands invaded by PA, and six uninvaded stands far from the river but located in the same region as the invaded sites (Fig. 1A). The invaded sites were selected based on a previous survey performed in 2013, and we selected sites that were only invaded by either PA or PU (Redondo et al. 2015). The uninvaded sites were located 1 to $3 \mathrm{~km}$ inland from the river, and the alders at these sites were thoroughly checked to confirm that they did not display symptoms of crown defoliation and stem necrosis. One of the uninvaded sites (PU1) was located $>3 \mathrm{~km}$ away from the river because of the absence of closer uninvaded alder stands. Alders are open wind-pollinated self-incompatible trees (Steiner and Gregorius 1999) whose populations display very little geographical genetic structure (Mingeot et al. 2016). We therefore assumed that uninvaded populations could well represent the population of alders growing at the riverside before the invasion of the $P$. alni complex. In each location, cones were picked from six healthy looking alder trees (three adult and three young trees). Tree age category was estimated based on the diameter at breast height (dbh) and the thickness of the bark, and trees were classified as young $(\mathrm{dbh}<15 \mathrm{~cm})$ or old $(\mathrm{dbh}>$ $15 \mathrm{~cm}$ ). Cones were stored in separate open bags, dried at room temperature for 3 months, and then opened to collect seeds. Seeds were stored inside sealed bags at $5^{\circ} \mathrm{C}$ for at least 3 weeks before germination. In total, cones were collected from 72 mother trees (18 mother trees from PU invaded sites, 18 mother trees from PA invaded sites, and 36 mother trees from uninvaded sites) growing at 12 different sites.

A subset of 200 seeds per mother tree was weighed to control for possible effects of seed weight on the seedling survival rate. For germination, seeds were surface sterilized by immersion in $1 \%$ sodium hypochlorite for $5 \mathrm{~min}$ and then planted in 24-well plastic racks filled with sterile vermiculite. The racks were kept in a plant growth chamber for 3 weeks under controlled conditions $\left(20^{\circ} \mathrm{C}, 16-\right.$ $\mathrm{h}$ photoperiod). Seedlings were watered every $48 \mathrm{~h}$ with a nutrient solution (Supplementary Table S1). After 3 weeks, the racks were inundated with distilled water to detach the vermiculite substrate from the seedlings without damaging the roots. From these seedlings, 60 similarly sized seedlings per mother tree (hereinafter progenies) were selected for the inoculation experiment.

Inoculation experiment. The progenies of the 72 mother trees were inoculated with zoospore solutions of two isolates of PA and two isolates of PU. The zoospore solution was prepared by immersing 20 well-colonized plugs $(7 \mathrm{~mm} \varnothing)$ of V8 agar medium (Erwin and Ribeiro 1996) into Erlenmeyer flasks containing $200 \mathrm{ml}$ of autoclaved liquid V8 media (20\% V8 juice, $80 \%$ distilled water). The flasks were incubated at $20^{\circ} \mathrm{C}$ in darkness for 7 days. After that period, the liquid V8 media were discarded and $200 \mathrm{ml}$ of autoclaved river water was added to the plugs to induce sporulation. River water was replaced every $12 \mathrm{~h}$ for 2 days. After 2 days, the autoclaved river water was replaced with sterile water and the flasks were incubated overnight at $20^{\circ} \mathrm{C}$ in darkness to induce zoospore release. The zoospore solution was adjusted to $3.5 \times 10^{4}$ zoospores $\mathrm{ml}^{-1}$ for inoculation.

The progenies of the 72 mother trees were inoculated with either (i) a zoospore solution of the pathogen present at the site where the seeds had been collected (PA or PU) (20 seedlings per progeny, 10 seedlings per isolate $\times 2$ isolates), (ii) a zoospore solution of the other pathogen species (20 seedlings per progeny, 10 seedlings per isolate $\times 2$ isolates), or (iii) a control inoculation of sterile water ( 20 seedlings per progeny). In total, 4,320 seedlings were inoculated. The inoculation procedure was performed in autoclaved empty $200-\mu l$ pipette tip boxes. Seedlings were inserted into the holes of the pipette tip rack so that the root system remained below the rack and in contact with the spore solution and the stem and leaves were above the rack (Fig. 1B). Progenies from the same mother tree were randomly assigned to a row in the rack. Inoculated seedlings were immersed in the zoospore solution for $2 \mathrm{~h}$. Control seedlings were immersed in sterile water.

After $2 \mathrm{~h}$, the seedlings were transferred to a rack containing sterile water to avoid desiccation before being placed in individual modified black Eppendorf tubes filled with distilled water, the lids of which were perforated to prevent the aerial parts from submerging in the water (Fig. 1C). Approximately $700 \mu \mathrm{l}$ of sterile water was added to each tube daily for 10 days with a pipette. The pipette tip was discarded after filling each Eppendorf tube. The health of each of the seedlings was assessed daily for the first 10 days postinoculation (dpi). Seedlings were recorded as healthy $(\mathrm{H})$ if leaves did not display any desiccation or wilting symptoms, symptomatic (S) if leaves displayed desiccation or wilting symptoms, and dead (D) if the aerial part of the seedling was necrotic and/or dry (Fig. 1C). For the progeny of each mother tree, each day for $10 \mathrm{dpi}$, we recorded the proportion of seedlings that 
survived and we also determined the proportion of the survival area under the curve $\left(\mathrm{AUC}_{\mathrm{j}}\right)$, which was calculated using the average of survival each day over the 10 days for each progeny, for each pathogen inoculation (equation 1):

$$
\mathrm{AUC}_{\mathrm{j}}=\frac{\sum_{\mathrm{i}=1}^{\mathrm{i}=10} \overline{\mathrm{S}}_{\mathrm{ij}}}{10}
$$

where $\bar{S}_{\mathrm{ij}}$ is the average survival of the seedlings of each progeny (j) at $i$ dpi. At the end of each inoculation, the root system of four dead seedlings per progeny $\times$ Phytophthora species combination (i.e., 576 seedlings in total) was excised, surface sterilized with ethanol for $10 \mathrm{~s}$, and plated onto Phytophthora-selective corn meal agar-PARPBH medium (Jeffers and Martin 1986) to reisolate the pathogen. When seedlings from the control experiment died, reisolation was also attempted to test for cross-contamination.

Statistical analysis. For the statistical analysis, we separated the data set of the progenies of the 72 mother trees into two groups, depending on whether the mother trees were growing at the PA invaded/uninvaded sites (36 mother trees) or at the PU invaded/ uninvaded sites (36 mother trees). The statistical analyses were performed separately in these two groups. We first tested the effect of the isolate used in the inoculation experiment on the survival of invaded versus uninvaded alder populations. We analyzed this with a logistic regression, using the survival AUC and survival $10 \mathrm{dpi}$ as the response and isolate $\times$ invaded/uninvaded as the explanatory variable. Because this interaction was not significant for either of the two species pathogens, we averaged the survival values of each progeny for both isolates and used these values in downstream statistical analyses. We tested the effect of the invaded/uninvaded origin of the seedling on survival $10 \mathrm{dpi}$ and the AUC of the inoculated seedlings with a logistic regression, using the proportion of survival seedlings $10 \mathrm{dpi}$ or the AUC as response variables, the invaded/ uninvaded origin, the age of the mother tree (young/old), and the region (PU1 to PU3, and PA1 to PA3; see Fig. 1) as explanatory variables. We created density histograms and boxplots of the distribution of AUC and survival $10 \mathrm{dpi}$ for the progenies from the invaded/uninvaded sites. All statistical analyses were performed with R software.

Narrow sense heritability. We estimated the narrow sense heritability $\left(\mathrm{h}^{2}\right)$ of susceptibility to PA and PU for each of the alder populations in invaded and uninvaded sites by fitting a logistic model using the days that a seedling remained alive as a response variable and the family (i.e., progeny from the same mother tree) as a random explanatory factor. We estimated $h^{2}$ as the variance component of the family divided by the total variance with the

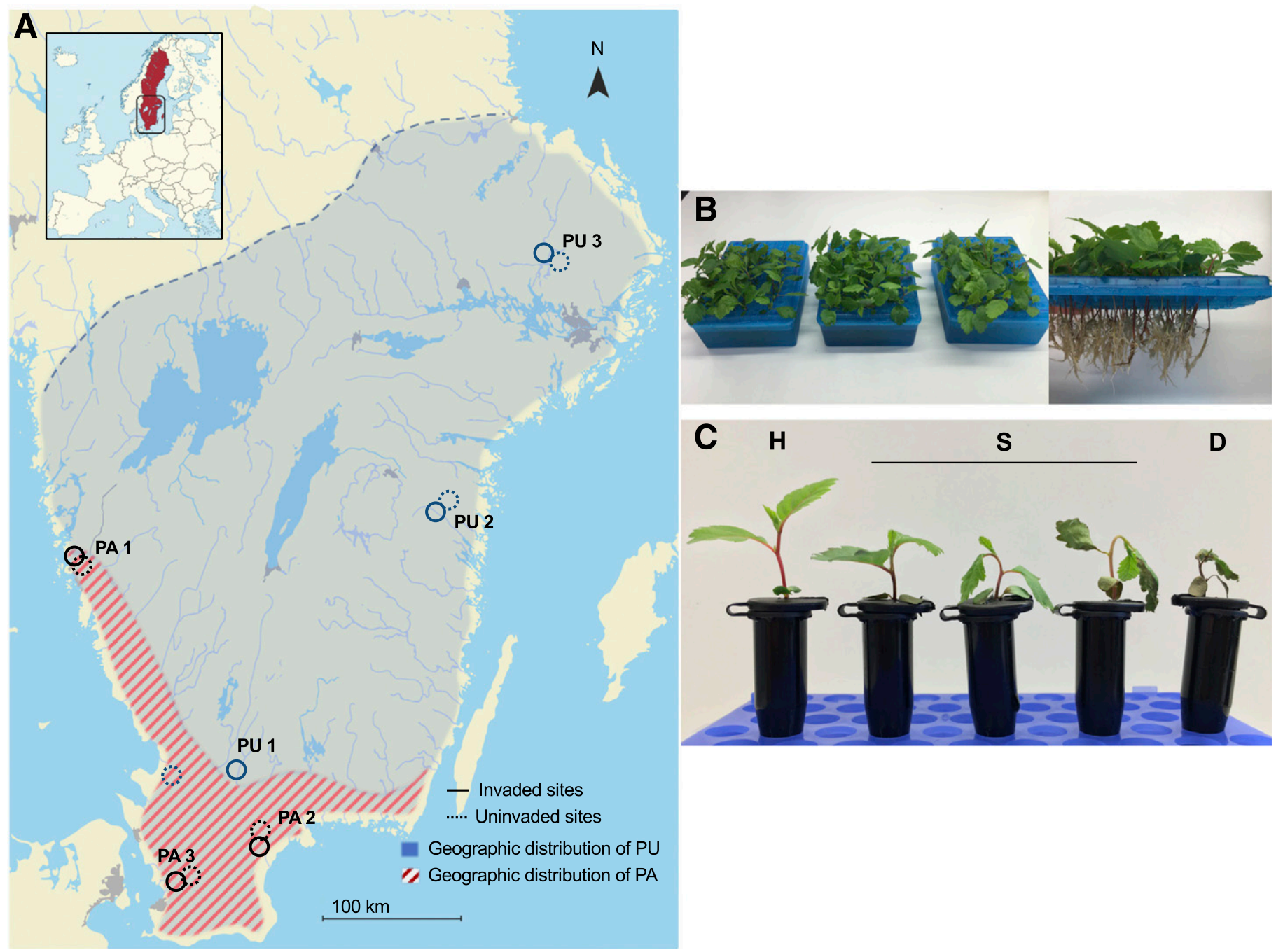

Fig. 1. Site locations and experimental setup used to inoculate alder seedlings. A, Location of the invaded and uninvaded sites for each of the three regions where Phytophthora $\times$ alni (PA) or P. uniformis (PU) is present. The geographic distribution of PU and PA is based on the survey of Redondo et al. (2015), which covered the shaded area up to the upper dashed line. B, Seedlings of each genotype randomly placed in rows of empty pipette tip boxes with their root systems immersed in a zoospore solution of PA or PU. C, Inoculated seedlings growing inside black Eppendorf tubes with a slot in the lid to enable water to be added daily to prevent desiccation. The health status of the seedlings was recorded daily during the first 10 days after inoculation. $\mathrm{H}=$ healthy seedling, $\mathrm{S}=$ symptomatic seedling, and $\mathrm{D}$ $=$ dead seedling. 
r.squaredGLMM function of the MuMIn package for R (Barton 2019). Because alder individuals of each family were not genetically identical but are likely to represent half-siblings in outcrossing A. glutinosa, the variance component of the family would give a measure of one-quarter of the additive genetic variance. We tested whether the $\mathrm{h}^{2}$ of the six uninvaded populations was different to PA and to PU with a logistic regression.

Simulated increase of survival AUC. We simulated in R a shift in the average survival AUC of a naive alder population over time as a consequence of the introduction of either PA or PU. To perform this simulation, we first obtained three parameters from the inoculation experiment: (a) the beta distribution of the survival AUC of naive genotypes when inoculated with PA and PU. From this distribution, we obtained the shape parameters with the function ebeta of the EnvStats package (Millard 2018); (b) the aggressiveness of PU ( $\left.\mathrm{A}_{\mathrm{PU}}\right)$ and $\mathrm{PA}\left(\mathrm{A}_{\mathrm{PA}}\right)$, calculated as 1 - AUC, where AUC is obtained from equation 1; and (c) the $h^{2}$ of the susceptibility of naïve populations to PA and PU.

We coded a loop in $\mathrm{R}$ software (the script is available in Supplementary Data File S1) to simulate an initial alder tree population of 1,000 individuals (SP) that was subjected to a continuous series of 120 death and seedling recruitment events caused by a hypothetical invasion of either PA or PU. The initial alder tree population was obtained by using the function rbeta of the EnvStats package (Millard 2018), using the two shape values obtained in parameter (a) from the inoculation experiment. Each simulated individual therefore had an AUC value, which corresponds to its susceptibility value (i.e., low AUC values mean high susceptibility and vice versa). In each event, we set a constant mortality rate for $\mathrm{PU}\left(\mathrm{M}_{\mathrm{PU}}\right)$ simulation of $5 \%$ of total standing trees for each event and a proportional mortality rate for the PA $\left(\mathrm{M}_{\mathrm{PA}}\right)$ simulation based on its higher level of aggressiveness $\left(\mathrm{M}_{\mathrm{PA}}=\right.$ $5 \% \times \frac{A_{\mathrm{PA}}}{\mathrm{A}_{\mathrm{PU}}}$. We used a constant recruitment rate $(\mathrm{R})$ of seedlings of $5 \%$ of total standing trees for every event for both PA and PU simulations. Each event in the loop consists of three steps. In the first step, we selected the amount of mortality that is genetically determined and therefore associated with a susceptibility (AUC) value. In this step, we used the $\mathrm{h}^{2}$ value, the parameter (c) of the inoculation experiment. For instance, $\mathrm{a} \mathrm{h}^{2}$ value of 0.3 would indicate that $30 \%$ of the dying trees would occur associated with genetic factors, whereas $70 \%$ occurs as a result of other factors and is therefore not associated with any AUC value. By doing so, we obtain the total mortality after the invasion of the pathogen as follows (step 1; equation 2):

$$
\mathrm{M}_{\mathrm{t}}=\mathrm{M} \times\left(1-\mathrm{h}^{2}\right) \times \mathrm{SP}_{\mathrm{R}}+\mathrm{M} \times \mathrm{h}^{2} \times \mathrm{SP}_{\mathrm{Q}}
$$

where $\mathrm{M}_{\mathrm{t}}$ is the total mortality after the invasion of PA or PU, M is the mortality rate of $\mathrm{PA}$ or $\mathrm{PU}, \mathrm{h}^{2}$ is the heritability to $\mathrm{PA}$ or $\mathrm{PU}, \mathrm{SP}_{\mathrm{R}}$ is the standing simulated population arranged at random, and $\mathrm{SP}_{\mathrm{Q}}$ is the standing simulated population arranged based on AUC values from lowest to highest. Note that in the first event of the simulation, the SP is the initial population of 1,000 individuals. After selecting the trees that died in step 1, the standing population is as follows (equation 3):

$$
\mathrm{SP}_{\mathrm{i}}=\mathrm{SP}-\mathrm{M}_{\mathrm{t}}
$$

In a second step, we recalculated the shape of the beta distribution of the standing population $\left(\mathrm{SP}_{\mathrm{i}}\right)$ and based on the recruitment rate (R), we obtained the new individuals that will be incorporated to the standing population with the rbeta function. Therefore, the total standing population after the first death and recruitment is as follows (step 2; equation 4):

$$
\mathrm{SP}_{\mathrm{j}}=\mathrm{SP}_{\mathrm{i}}+\mathrm{R} \times \mathrm{SP}_{\mathrm{i}}
$$

In the third step of each event, we obtained the average AUC of the standing population after the recruitment $\left(\mathrm{SP}_{\mathrm{j}}\right)$ and the relative increase of $\mathrm{AUC}(\% \Delta \mathrm{AUC})$ compared with the initial population as follows (step 3; equation 5):

$$
\% \Delta \mathrm{AUC}=\frac{\mathrm{AUC}\left(\mathrm{SP}_{\mathrm{j}}\right)-\mathrm{AUC}(\mathrm{SP})}{\mathrm{AUC}(\mathrm{SP})}
$$

In a second phase, we reran the same simulation but this time accounting for the decrease in the mortality rate caused by PA as a result of the virulence trade-off hypothesis. For this, we first studied the effect that the different aggressiveness levels of PA and PU would have on the number of infectious trees and therefore on the inoculum pressure. To do so, we used a variation of the compartmental SIR model, in which we used different mortality rates for PA and PU. In this model, "recovered" trees that could not become infected again were considered as "dead" trees. The script for the SIR model was obtained from the website of the Theoretical Biology group in the University of Zurich Department of Environmental Systems Science (https://www.tb.ethz.ch/) and was implemented in the R environment. We ran the SIR on a simulated population of 500 alders over 100 events. For PU, we used the default parameter for infectivity $(\beta=0.001)$ and constant mortality rate $(r=0.1)$. For PA, we used the same value for $\beta$ and increased the value of $\mathrm{r}$ proportionally to its higher aggressiveness ( $\mathrm{r}$ for PA $=$ 0.13 ). We determined the decrease in the number of infectious trees in the SIR simulation for PA compared with that for PU, and this ratio was assumed to be proportional to the decrease in the PA inoculum pressure (i.e., $10 \%$ fewer infectious trees, approximately $10 \%$ fewer zoospores). To test how this ratio was influenced by the parameters of the SIR, we ran the SIR model again giving different values for each of the parameters $\beta$ and $\mathrm{r}$, increasing them with 10 , 20,50 , and $100 \%$ while keeping the other parameter constant. Increases in $\beta$ resulted in an increase of the ratio of infectious trees between PA and PU of 0.38 to $1.2 \%$. Increases in $r$ resulted in a decrease of the ratio of infectious trees between PA and PU of 0.52 to $8.9 \%$ (Supplementary Table S2).

To test the effect of a decrease in inoculum pressure on the mortality rate of the population, we assumed that mortality would follow a general dose-response curve, which we fitted using a general three-parameter logistic equation (equation 6) obtained from the curve fitting guide of GraphPad software (https:// www.graphpad.com):

$$
Y=\min +\frac{(\min -\max )}{1+10^{\operatorname{LogEC}} 50-x}
$$

where $\mathrm{Y}$ is the response (mortality), min is $0 \%$ mortality, max is $100 \%$ mortality, and $\operatorname{LogEC}_{50}$ is the logarithm of the effective concentration of inoculum (i.e., the $\mathrm{X}$ value when the response is halfway between the bottom and top). We subsequently determined the proportional decrease in mortality as a consequence of the proportional decrease in $\mathrm{EC}_{50}$. Based on this, we corrected the mortality rate of PA and reran the first simulation, keeping the rest of the simulation parameters constant.

\section{RESULTS}

Differences in survival between invaded and uninvaded sites. A significant difference in terms of survival between seedlings from invaded and uninvaded areas was observed for PU but not for PA (Table 1). Survival in PU invaded sites was higher than that in uninvaded sites (AUC: $66.8 \%$ versus $56.5 \% ; P=0.019$ ) (Fig. 2A). Survival from invaded sites 10 dpi was almost double the survival from uninvaded sites $(30.5 \%$ versus $15.9 \% ; P=0.008)$ (Fig. 2B). The histogram of the survival of the progenies showed that the most susceptible genotypes were present in uninvaded sites and absent in invaded sites (Fig. 2A and B). For PA, no survival 
differences between invaded and noninvaded sites were observed (Fig. 2C and D); however, we found some geographical structure in terms of susceptibility (Table 1). PU invaded progenies seemed to be less susceptible to PA than progenies from trees from inland uninvaded sites (Supplementary Fig. S1 A and B). Within the area of distribution of PA, no differences in survival to PU between riverbank and inland progenies were observed (Supplementary Fig. S1C and D). All observed patterns appeared unrelated to the age of the mother trees.

Neither the AUC nor the survival rate of progeny 10 dpi with either PA or PU was correlated with seed weight $(P=0.88$ for PA, and $P=0.16$ for PU). PA and PU were reisolated from all of the dead seedlings inoculated with PA or PU, respectively. The survival rate of progenies that received the mock inoculation treatment was 98.7\% 10 dpi. Neither PA nor PU was reisolated from dead seedlings that received mock inoculations.

Differences in aggressiveness between PU and PA, and narrow sense heritability of susceptibility to PU and PA. PA was $27 \%$ more aggressive than PU (1 - AUC of 52 versus $41 \%$, respectively; $P<0.0001$ ) (Fig. $3 \mathrm{~A})$. The $\mathrm{h}^{2}$ of uninvaded populations to PU was significantly higher than to PA $(0.32$ versus $0.1 ; P=0.035)$ (Fig. 3B). The heritability of invaded populations to PU and PA was not significantly different. No differences in terms of heritability between invaded and uninvaded sites were found within PU or PA regions, although PU invaded (and less susceptible) populations tended to have a lower heritability than those unexposed to the pathogen $(0.29$ versus $0.41 ; P=0.48)$.

Simulations of an increase of average survival of infected individuals after the invasion of PA and PU. The values of aggressiveness and heritability of uninvaded populations were used as the input for the simulation. PA was $27 \%$ more aggressive than PU, which, according to an SIR model, would result in a relative reduction in the number of infectious trees in the epidemic of $24 \%$ (9.9 versus $7.5 \%$ ) (Supplementary Fig. S2). Assuming that mortality follows a dose-response curve, the mortality in the population caused by PA after accounting for an inoculum decrease would be $14 \%$ lower than that predicted by the model that did not account for a virulence trade-off (i.e., the mortality rate in the simulations with and without accounting for the virulence trade-off would be 5.4 versus $6.3 \%$, respectively).

In simulations, $\mathrm{h}^{2}$ was used as a proxy for selection by PA and PU that occurs as a result of genetic or stochastic factors; for example, a $\mathrm{h}^{2}$ value of 0.32 was used in the PU simulation to indicate that $32 \%$ of tree deaths would be attributable to genetic factors and $68 \%$ would be stochastic. When only considering the effect of random versus nonrandom killing of trees, PU will produce a faster gain in survival than PA (25.6 versus $13.8 \%$ for PU and PA after 120 generations, respectively; Fig. 4), mirroring what was observed in the field. It would take approximately three times as many generations to obtain a similar gain in AUC to PA as to PU (Fig. 4). When we used the mortality rate values modified by the trade-off hypothesis instead of the original mortality rate values, a very small overall impact on the development of AUC was observed (i.e., 12.9 versus $13.8 \%$ gain in AUC in PA with and without considering tradeoff hypothesis after 120 generations) (Fig. 4).
Altogether, our simulations indicated that a gain in AUC (i.e., overall survival) seems to be more impaired by a low level of genetic variation in susceptibility in the tree population than by a possible effect of the aggressiveness of the pathogen limiting its transmission (virulence trade-off hypothesis).

\section{DISCUSSION}

Theory predicts that naive host populations may adapt to a new pathogen if the population is of sufficient size and has sufficient genetic variability for natural selection to act (Parker and Gilbert 2004; Strauss et al. 2006). Following the introduction and spread of exotic species of the P. alni complex during the 1990s, we looked for signs of natural selection along alder stands growing in river banks and exposed to either PU or PA. We found indications that PU may have been able to shift the genetic profile of the host, but this did not seem to be the case for PA. Progenies of trees exposed to PU showed higher survival than uninvaded progenies from trees growing inland. The most susceptible genotypes (AUC $<40 \%$ ) were only found in uninvaded sites, whereas the most resistant (AUC $>80 \%$ ) were found in invaded sites. Our observations could fit well into a scenario in which PU would have eliminated the susceptible genotypes in invaded sites, thus explaining the increase of average survival of invaded populations. We speculate that the lack of natural selection in invaded PA sites could be attributable to the lower levels of initial genetic variation in susceptibility to PA than to PU. Both the increase of average survival in PU invaded sites and the higher $\mathrm{h}^{2}$ of naive alder populations to PU suggest that alder populations in Sweden may have a higher potential to adapt to PU than to PA. The levels of heritability of susceptibility to PU in our study are similar to or higher than those of other novel pathosystems such as Fraxinus excelsior to Hymenoscyphus fraxineus (Kjær et al. 2012; Pliura et al. 2015) or Ulmus spp. to O. novo-ulmi (Solla et al. 2014).

Simulation data seem to support a key role of native genetic resistance in the host population to adapt to a new pathogen. We used the values of heritability of naive alders as a proxy of selection pressure associated with genetic factors in our simulations. Low values of heritability indicate little variation in survival between half-sibling families. Therefore, in a scenario with low values of heritability, variation in mortality rates after pathogen invasion could be explained by environmental factors or stochasticity. Following that logic, we found that naive alder populations would need three times more generations to adapt to PA than to PU, which could explain the observed lack of differences in survival after PA inoculation between invaded/uninvaded populations. Nevertheless, the results of our simulation do not have predictive power beyond this case study. Some of the parameters of the simulation, such as the constant mortality rates and recruitment of seedlings kept over time after a simulated invasion, may not mirror the consequences of a Phytophthora invasion in nature. Other aspects such as climatic factors, which seem to play a role in the severity of the attacks posed by P. alni (Aguayo et al. 2014; Černý et al. 2012; Redondo et al. 2015), were not included in our simulations.

TABLE 1. Summary of the statistics of the inoculation experiment in which progenies of alder mother trees obtained from regions with Phytophthora uniformis $(\mathrm{PU})$ and Phytophthora $\times$ alni $(\mathrm{PA})$ were inoculated with the pathogen species present in those regions ${ }^{\mathrm{a}}$

\begin{tabular}{|c|c|c|c|c|c|c|c|c|c|c|c|c|}
\hline \multirow[b]{3}{*}{ Variable } & \multicolumn{6}{|c|}{ Progenies from regions with PU inoculated with PU } & \multicolumn{6}{|c|}{ Progenies from regions with PA inoculated with PA } \\
\hline & \multicolumn{3}{|c|}{ Survival AUC } & \multicolumn{3}{|c|}{ Survival $10 \mathrm{dpi}$} & \multicolumn{3}{|c|}{ Survival AUC } & \multicolumn{3}{|c|}{ Survival $10 \mathrm{dpi}$} \\
\hline & df & $F$ value & $P$ value & df & $F$ value & $P$ value & df & $F$ value & $P$ value & df & $F$ value & $P$ value \\
\hline Invaded/uninvaded site & 1 & 5.52 & 0.025 & 1 & 7.31 & 0.011 & 1 & 1.053 & 0.31 & 1 & 0.0082 & 0.93 \\
\hline Age of the mother tree & 1 & 0.008 & 0.93 & 1 & 0.015 & 0.90 & 1 & 0.75 & 0.39 & 1 & 0.65 & 0.43 \\
\hline Region & 2 & 0.28 & 0.75 & 2 & 0.16 & 0.85 & 2 & 4.89 & 0.014 & 2 & 3 & 0.064 \\
\hline Residuals & 31 & - & - & 31 & - & - & 31 & - & - & 31 & - & - \\
\hline
\end{tabular}

a $\mathrm{AUC}=$ area under the curve, $\mathrm{dpi}=$ days postinoculation, and $\mathrm{df}=$ degrees of freedom. Significant $P$ values $(<0.05)$ are displayed in bold. 
The virulence trade-off hypothesis could offer an alternative explanation for the lack of natural selection observed in populations invaded by PA compared with those invaded by PU. According to this theory, the greater aggressiveness of PA compared with that of PU would produce short-duration infections, which would reduce the transmission of the pathogen (Alizon et al. 2009). We found little support for this alternative explanation. Based on aggressiveness data from the in vitro experiment, we simulated the differences between PA and PU in terms of the number of infectious trees, the inoculum pressure, and the gain of AUC after a putative invasion by PA and PU. Our simulation provided a very simplified model of the invasion; nevertheless, it seemed to show that the penalty for being exposed to a more aggressive pathogen such as PA would be of an order of magnitude smaller than the effect of having little genetic variation in susceptibility. These findings might not reflect a general pattern for all invaders, as the smaller impact of aggressiveness may be attributable to the moderate differences between PA and PU found in our in vitro system. Our aggressiveness in vitro seemed to
A

$$
\begin{aligned}
& \text { Progenies from } \\
& \text { regions with PU }
\end{aligned}
$$

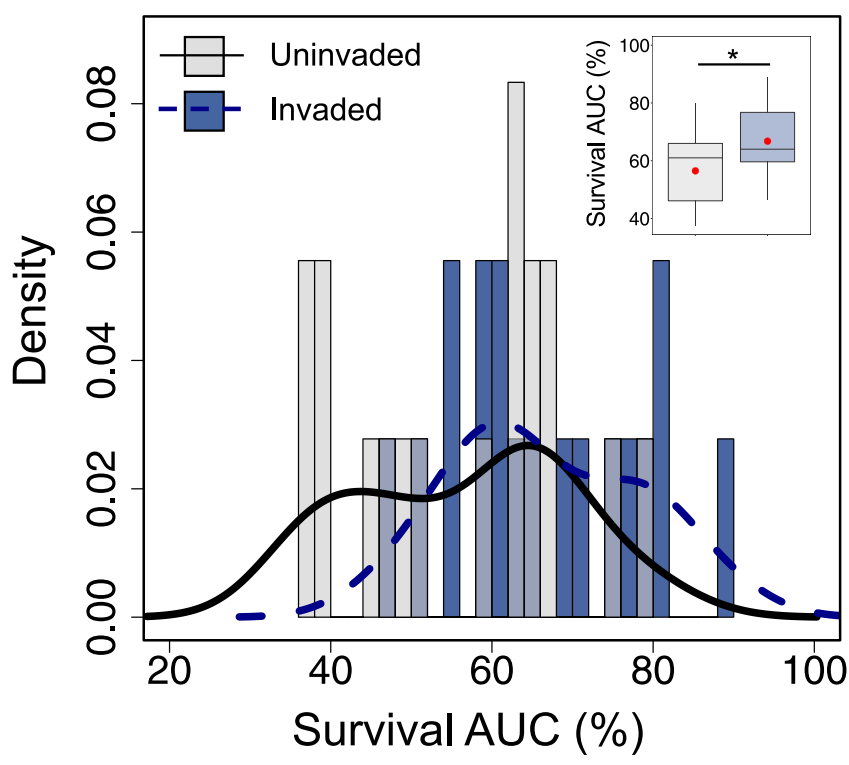

C

$$
\begin{aligned}
& \text { Progenies from } \\
& \text { regions with PA }
\end{aligned}
$$

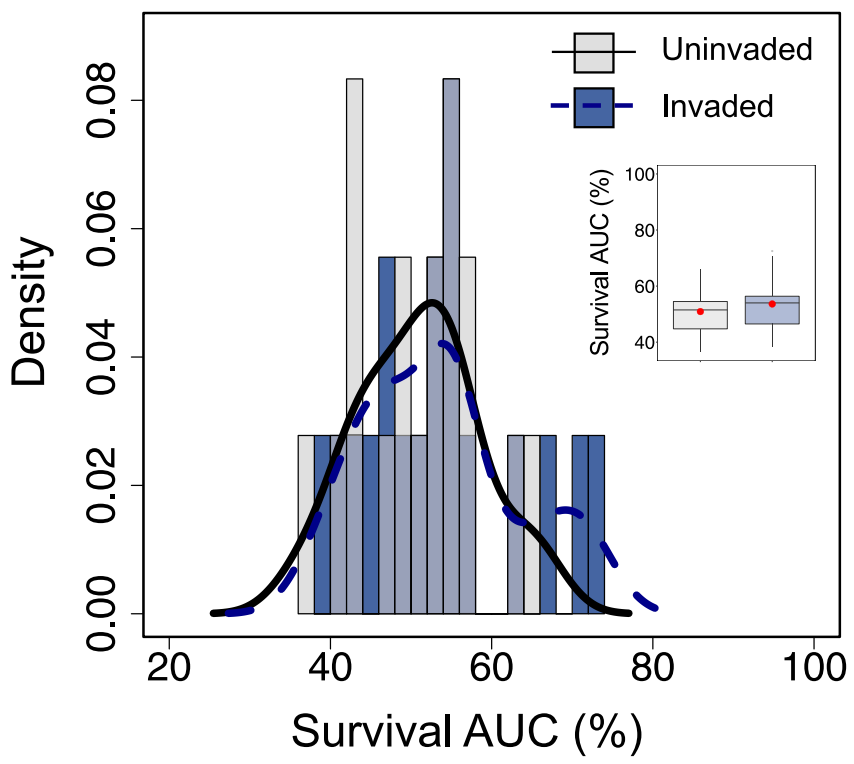

B
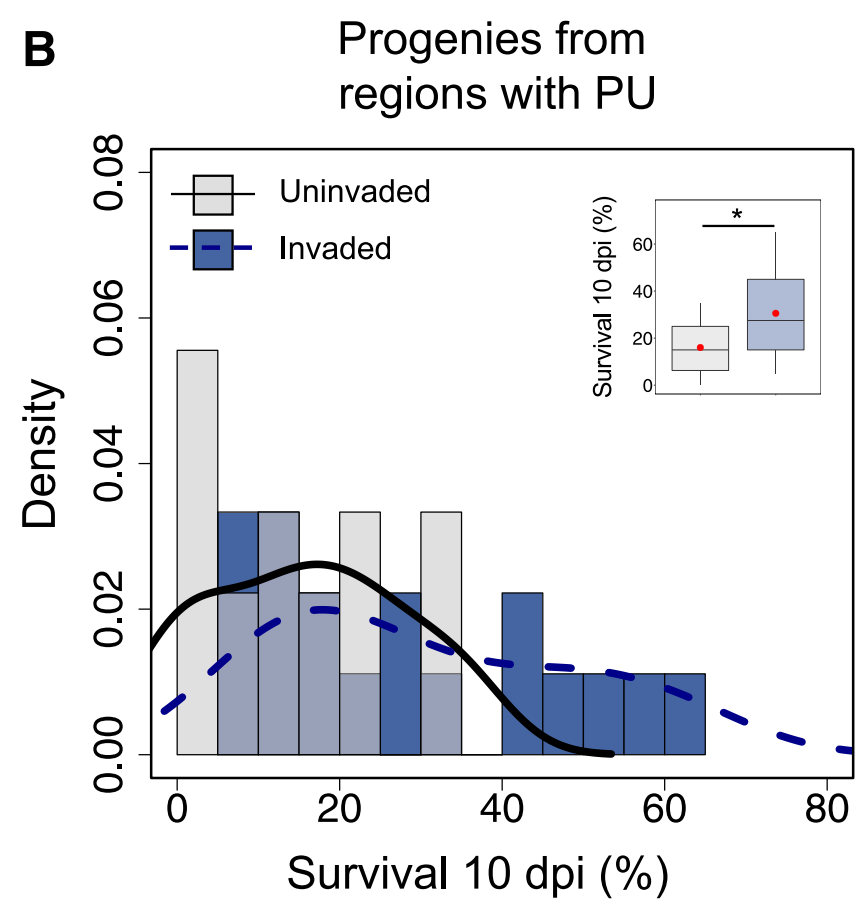

D

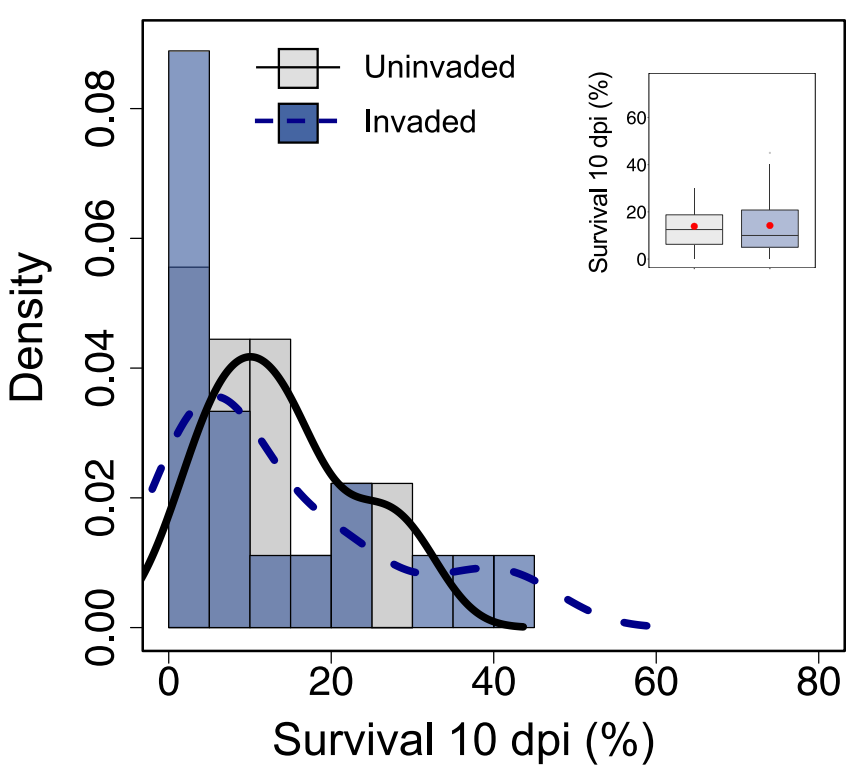

Fig. 2. Distributions of the survival of progenies of alder mother trees from Phytophthora uniformis (PU) and Phytophthora $\times$ alni (PA) regions used in the inoculation experiment. A and B, The histogram, density lines, and box plot of the area under the curve (AUC) and survival 10 days postinoculation (dpi), respectively, of alder progenies from regions with PU and inoculated with PU. C and D, The histogram, density lines, and box plot of the AUC and survival 10 dpi, respectively, of alder progenies from regions with PA and inoculated with PA. Dots on the box plots depict the averages for each group. Asterisks indicate significant differences at $P<0.05$. 
mirror the aggressiveness in the field, where a similar proportion of diseased trees in sites infected by PA and PU was found (Redondo et al. 2015). Nevertheless, Montarry et al. (2007) did not find evidence of trade-off between virulence and inoculum survival of $P$. infestans, even though the differences in lesion length caused by the most and least aggressive strains were twofold. Even if we applied those values in our simulation (i.e., the aggressiveness of PA being twice the one from PU), the role of the trade-off hypothesis would be of a much lesser importance that genetic variation in our simulated system (results not shown). The negative effect of high aggressiveness on transmission rate could also be dependent on the timing of the invasion at a regional scale: a widespread availability of susceptible trees may allow aggressive pathogens to have similar transmission rates than less aggressive ones. The effect of the tradeoff hypothesis also depends on the life history of the pathogen. It could be that in polycyclic diseases such as those of Phytophthora spp., faster infections can lead to faster resource uptake from the host, higher sporulation rates, and therefore higher transmission. This would imply that the more aggressive PA could produce higher amounts of inoculum and colonize susceptible trees in a shorter time span than the less aggressive PU. This could explain why PA seems to dominate in areas where conditions are favorable for both species (Redondo et al. 2015), pointing to a certain competitive advantage of PA over PU. Similarly, other aggressive forest pathogens such as O. novo-ulmi have been able to displace their less aggressive counterparts (Brasier and Buck 2001).

A third alternative explanation for differences in the natural selection of alder by PU and PA could be a much earlier arrival of PU in Sweden compared with that of PA. However, both pathogens were reported for the first time at once in Sweden during the early 1990s (Olsson 1999). The cross-inoculation also seems to discard a later arrival of PA, since we did not find signs that riverbank alders in the areas currently occupied by PA had been previously selected by PU. The higher survival of alders in riverbanks could also be the result of natural selection exerted by other Phytophthora species commonly found in Swedish river streams (Redondo et al. 2018a,

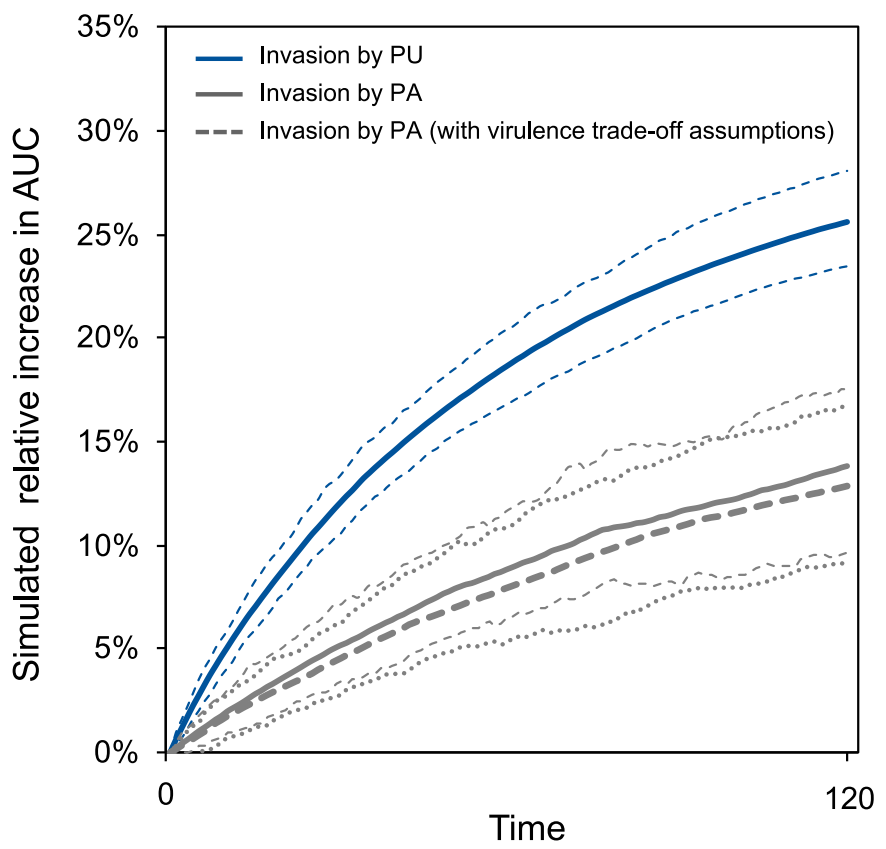

Fig. 4. Simulations of a shift in the average survival of a naïve alder population over time as a consequence of invasion by either Phytophthora $\times$ alni (PA) or P. uniformis (PU). The solid black (blue) line (PU invasion) and solid gray line (PA invasion) represent the mean relative increase in alder survival area under the curve (AUC) between an initial naïve population that had not been exposed to the pathogen as well as the population after each of the 120 simulated selection events exerted by PA and PU, respectively, without considering the assumptions of the trade-off hypothesis for PA. The dashed thin lines represent the $95 \%$ confidence interval $(95 \% \mathrm{CI})$ of the mean. The short dashed gray line (PA invasion) represents the mean relative increase in survival AUC, accounting for the assumptions of the trade-off hypothesis. The dotted lines represent the $95 \%$ CI of the mean.
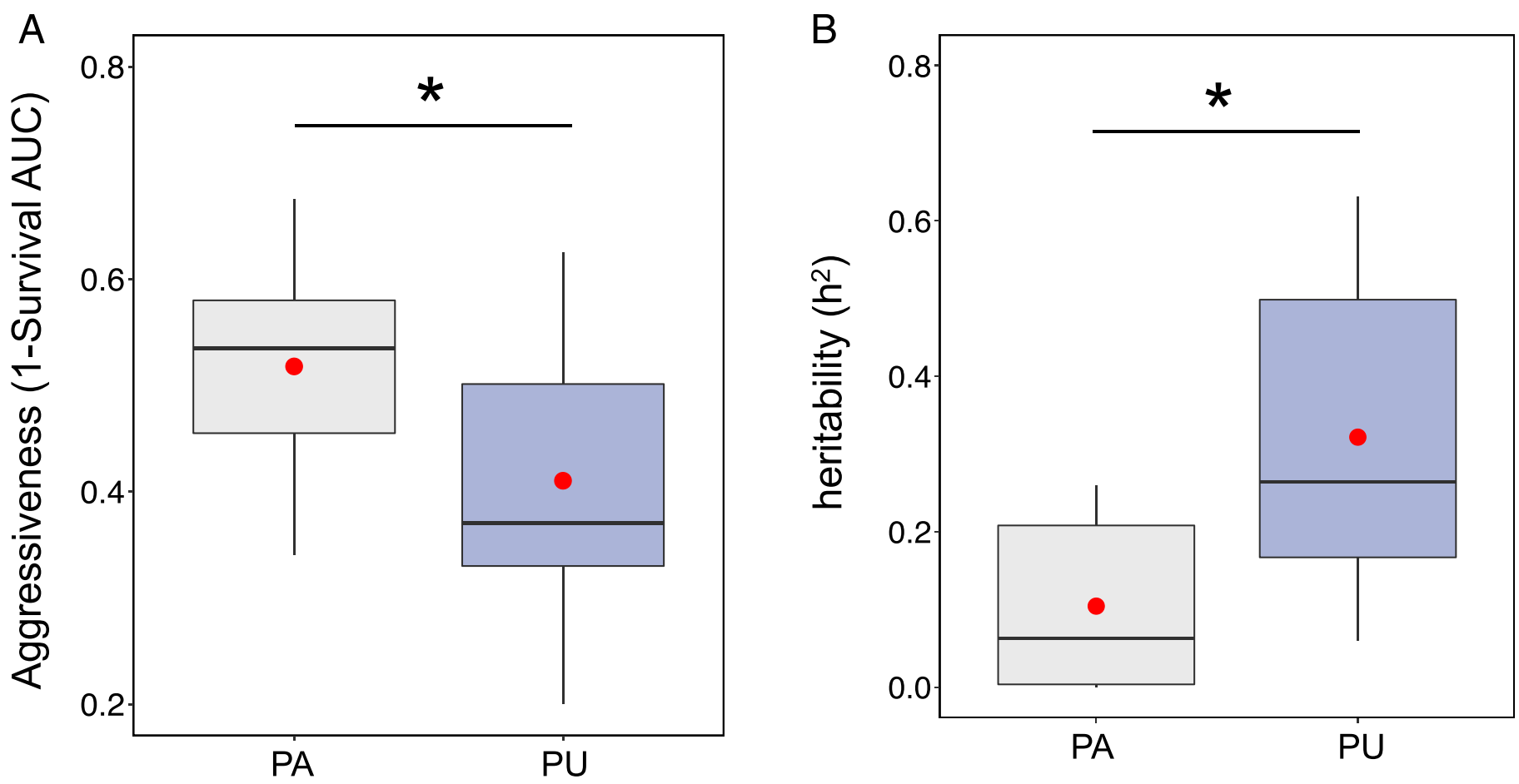

Fig. 3. Response of families from uninvaded sites to Phytophthora $\times$ alni (PA) and P. uniformis (PU). A, Differences in aggressiveness between PU and PA. Only the 36 alder progenies from the six uninvaded sites were included in this analysis. B, Differences in narrow sense heritability $\left(\mathrm{h}^{2}\right)$ of the half-sibling populations $(n=6)$ of alders from uninvaded sites when inoculated with PA and PU. Dots on the box plots depict the averages for each group. Asterisks indicate significant differences at $P<0.05$. AUC $=$ area under the curve. 
b). However, Phytophthora spp. seem to be present in all rivers, and it seems unlikely that the selection happened only on PU invaded sites. Finally, we cannot discard a scenario in which resistance to PA would be monogenic and rare and therefore undetectable with the number of screened progenies. In other invasive pathogens, such as white pine blister rust (Cronartium ribicola), resistance can be as rare as 0.001 in the naive host population (Sniezko et al. 2014). To confirm whether monogenic resistance is present, a much deeper screening of families should be carried out (i.e., in the order of thousands) (Sniezko et al. 2014).

In our study, we explained a difference in survival observed in nature, based on in vitro inoculations, which may not represent the conditions in the field. Nevertheless, in the case of other Phytophthora species, the susceptibility levels of trees inoculated under field conditions and of those inoculated in vitro were significantly correlated (Dodd et al. 2005). We performed our inoculations using zoospore suspensions because this was previously shown to be the best method to screen for susceptibility of black alder to PA (Chandelier et al. 2016). Moreover, inoculation based on zoospores was the method that best correlated the development of the disease in vitro with the susceptibility of the host in the field for 39 plant species (Hansen et al. 2005).

In conclusion, we showed that selection for lower susceptibility to a new forest pathogen may occur under natural conditions. This is one of the many steps for a host to adapt to a new pathogen. In light of our results, it seems that a lack of genetic variation could pose a stronger impediment (Altizer et al. 2003; Budde et al. 2016; Parker and Gilbert 2004; Strauss et al. 2006), than epidemiological aspects related to the aggressiveness of the pathogen (Alizon et al. 2009). Our findings suggest that given that the new pathogen reduces the host's fitness, natural selection will occur if genetic variation in susceptibility exists (high $\mathrm{h}^{2}$ ), as we observed for PU. If the new pathogen finds low genetic variation in susceptibility within the naive host population, natural selection will be minimal, as observed for PA. Without natural selection, shortterm adaptation is unlikely to occur naturally. Our findings open the door to the use of heritability as a predictor for the likelihood of natural adaptation of naive tree populations to invasive pathogens and therefore can be useful for risk assessment purposes or when managing pathogen invasions.

\section{ACKNOWLEDGMENTS}

We acknowledge the kind help of Maria Jonsson during the laboratory work and the help of Malin Elfstrand and particularly Johanna Boberg during the design of the experiment. We acknowledge the constructive comments of the two anonymous referees on a previous version of this manuscript.

\section{LITERATURE CITED}

Aguayo, J., Elegbede, F., Husson, C., Saintonge, F.-X., and Marçais, B. 2014. Modeling climate impact on an emerging disease, the Phytophthora alniinduced alder decline. Glob. Change Biol. 20:3209-3221.

Alizon, S., Hurford, A., Mideo, N., and Van Baalen, M. 2009. Virulence evolution and the trade-off hypothesis: History, current state of affairs and the future. J. Evol. Biol. 22:245-259.

Altizer, S., Harvell, D., and Friedle, E. 2003. Rapid evolutionary dynamics and disease threats to biodiversity. Trends Ecol. Evol. 18:589-596.

Barton, K. 2019. Package "MuMIn." Multi-model inference, version 1.43.6. https://cran.r-project.org/web/packages/MuMIn/MuMIn.pdf

Bjelke, U., Boberg, J., Oliva, J., Tattersdill, K., and McKie, B. G. 2016. Dieback of riparian alder caused by the Phytophthora alni complex: Projected consequences for stream ecosystems. Freshw. Biol. 61:565-579.

Brasier, C. M. 1991. Ophiostoma novo-ulmi sp. nov., causative agent of current Dutch elm disease pandemics. Mycopathologia 115:151-161.

Brasier, C. M., and Buck, K. W. 2001. Rapid evolutionary changes in a globally invading fungal pathogen (Dutch elm disease). Biol. Invasions 3: 223-233.

Brasier, C. M., and Kirk, S. A. 2001. Comparative aggressiveness of standard and variant hybrid alder phytophthoras, Phytophthora cambivora and other
Phytophthora species on bark of Alnus, Quercus and other woody hosts. Plant Pathol. 50:218-229.

Brasier, C. M., Kirk, S. A., Delcan, J., Cooke, D. E. L., Jung, T., and Man In't Veld, W. A. 2004. Phytophthora alni sp. nov. and its variants: Designation of emerging heteroploid hybrid pathogens spreading on Alnus trees. Mycol. Res. 108:1172-1184.

Budde, K. B., Nielsen, L. R., Ravn, H. P., and Kjær, E. D. 2016. The natural evolutionary potential of tree populations to cope with newly introduced pests and pathogens-Lessons learned from forest health catastrophes in recent decades. Curr. For. Rep. 2:18-29.

Carroll, S. P., Jørgensen, P. S., Kinnison, M. T., Bergstrom, C. T., Denison, R. F., Gluckman, P., et al. 2014. Applying evolutionary biology to address global challenges. Science 346:1245993.

Černý, K., Filipová, N., and Strnadová, V. 2012. Influence of low temperature and frost duration on Phytophthora alni subsp. alni viability. For. Syst. 21: 337-342.

Chandelier, A., Husson, C., Druart, P., and Marçais, B. 2016. Assessment of inoculation methods for screening black alder resistance to Phytophthora $\times$ alni. Plant Pathol. 65:441-450.

Daszak, P., Cunningham, A. A., and Hyatt, A. D. 2000. Emerging infectious diseases of wildlife. Threats to biodiversity and human health. Science 287: 443-449.

De Castro, F., and Bolker, B. 2005. Mechanisms of disease-induced extinction. Ecol. Lett. 8:117-126.

Dodd, R. S., Hüberli, D., Douhovnikoff, V., Harnik, T. Y., Afzal-Rafii, Z., and Garbelotto, M. 2005. Is variation in susceptibility to Phytophthora ramorum correlated with population genetic structure in coast live oak (Quercus agrifolia)? New Phytol. 165:203-214.

Ennos, R. A. 2015. Resilience of forests to pathogens: An evolutionary ecology perspective. Forestry 88:41-52.

Erwin, D. C., and Ribeiro, O. K. 1996. Phytophthora Diseases Worldwide. American Phytopathological Society, St. Paul, MN.

Fisher, M. C., Henk, D. A., Briggs, C. J., Brownstein, J. S., Madoff, L. C., McCraw, S. L., et al. 2012. Emerging fungal threats to animal, plant and ecosystem health. Nature 484:186-194.

Griffin, G. J. 2000. Blight control and restoration of the American chestnut. J. For. 98:22-27.

Hansen, E. M., Parke, J. L., and Sutton, W. 2005. Susceptibility of Oregon forest trees and shrubs to Phytophthora ramorum: A comparison of artificial inoculation and natural infection. Plant Dis. 89:63-70.

Hendry, A. P., Gotanda, K. M., and Svensson, E. I. 2017. Human influences on evolution, and the ecological and societal consequences. Philos. Trans. R. Soc. B Biol. Sci. 372:20160028.

Hepting, G. H. 1974. Death of the American chestnut. J. For. Hist. 18:61-67.

Husson, C., Aguayo, J., Revellin, C., Frey, P., Ioos, R., and Marçais, B. 2015. Evidence for homoploid speciation in Phytophthora alni supports taxonomic reclassification in this species complex. Fungal Genet. Biol. 77:12-21.

Jeffers, S. N., and Martin, S. B. 1986. Comparison of two media selective for Phytophthora and Pythium species. Plant Dis. 70:1038-1043.

Jung, T., and Blaschke, M. 2004. Phytophthora root and collar rot of alders in Bavaria: Distribution, modes of spread and possible management strategies. Plant Pathol. 53:197-208.

Kjær, E. D., McKinney, L. V., Nielsen, L. R., Hansen, L. N., and Hansen, J. K. 2012. Adaptive potential of ash (Fraxinus excelsior) populations against the novel emerging pathogen Hymenoscyphus pseudoalbidus. Evol. Appl. 5: 219-228.

Kowalski, T. 2006. Chalara fraxinea sp. nov. associated with dieback of ash (Fraxinus excelsior) in Poland. For. Pathol. 36:264-270.

Kroll, R. E., and Eide, C. J. 1981. Effect of inoculum concentration of Phytophthora infestans on potato late blight. Am. Potato J. 58:153-161.

McCallum, H. 2012. Disease and the dynamics of extinction. Philos. Trans. R. Soc. B. 367:2828-2839.

Millard, S. P. 2018. Package "EnvStats." Package for environmental statistics, version 2.3.1. https://cran.r-project.org/web/packages/EnvStats/EnvStats.pdf

Mingeot, D., Husson, C., Mertens, P., Watillon, B., Bertin, P., and Druart, P. 2016. Genetic diversity and genetic structure of black alder (Alnus glutinosa [L.] Gaertn) in the Belgium-Luxembourg-France cross-border area. Tree Genet. Genomes 12:24.

Montarry, J., Corbiere, R., and Andrivon, D. 2007. Is there a trade-off between aggressiveness and overwinter survival in Phytophthora infestans? Funct. Ecol. 21:603-610.

Olsson, C. H. B. 1999. Diagnosis of root-infecting Phytophthora spp. Doctoral thesis, Swedish University of Agricultural Sciences, Uppsala, Sweden.

Parker, I. M., and Gilbert, G. S. 2004. The evolutionary ecology of novel plantpathogen interactions. Annu. Rev. Ecol. Evol. Syst. 35:675-700.

Pliura, A., Lygis, V., Marčiulyniene, D., Suchockas, V., and Bakys, R. 2015. Genetic variation of Fraxinus excelsior half-sib families in response to ash dieback disease following simulated spring frost and summer drought treatments. IForest 9:12-22. 
Redondo, M. A., Boberg, J., Olsson, C. H. B., and Oliva, J. 2015. Winter conditions correlate with Phytophthora alni subspecies distribution in Southern Sweden. Phytopathology 105:1191-1197.

Redondo, M. A., Boberg, J., Stenlid, J., and Oliva, J. 2018a. Contrasting distribution patterns between aquatic and terrestrial Phytophthora species along a climatic gradient are linked to functional traits. ISME J. 12: 2967-2980.

Redondo, M. A., Boberg, J., Stenlid, J., and Oliva, J. 2018b. Functional traits associated with the establishment of introduced Phytophthora spp. in Swedish forests. J. Appl. Ecol. 55:1538-1552.

Rizzo, D. M., Garbelotto, M., and Hansen, E. M. 2005. Phytophthora ramorum: Integrative research and management of an emerging pathogen in California and Oregon forests. Annu. Rev. Phytopathol. 43:309-335.

Santini, A., Ghelardini, L., De Pace, C., Desprez-Loustau, M. L., Capretti, P., Chandelier, A., et al. 2013. Biogeographical patterns and determinants of invasion by forest pathogens in Europe. New Phytol. 197:238-250.
Sniezko, R. A., Smith, J. A., Liu, J.-J, and Hamelin, R. C. 2014. Genetic resistance to fusiform rust in southern pines and white pine blister rust in white pines-A contrasting tale of two rust pathosystems-Current status and future prospects. Forests 5:2050-2083.

Solla, A., Bohnens, J., Collin, E., Diamandis, S., Franke, A., Gil, L., et al. 2005. Screening European elms for resistance to Ophiostoma novo-ulmi. For. Sci. 51:134-141.

Solla, A., López-Almansa, J. C., Martín, J. A., and Gil, L. 2014. Genetic variation and heritability estimates of Ulmus minor and Ulmus pumila hybrids for budburst, growth and tolerance to Ophiostoma novo-ulmi. IForest 8:422-430.

Steiner, W., and Gregorius, H. R. 1999. Incompatibility and pollen competition in Alnus glutinosa: Evidence from pollination experiments. Genetica 105: 259-271.

Strauss, S. Y., Lau, J. A., and Carroll, S. P. 2006. Evolutionary responses of natives to introduced species: What do introductions tell us about natural communities? Ecol. Lett. 9:357-374. 